University of Wollongong

Research Online

Faculty of Engineering - Papers (Archive)

Faculty of Engineering and Information

Sciences

2002

\title{
CoALA-SPECT: a coded aperture laboratory animal SPECT system for pre clinical imaging
}

S. R. Meikle

Royal Prince Alfred Hospital, Sydney

R. Wojcik

Thomas Jefferson National Accelerator Facility, Virginia, USA

A. G. Weisenberger

Thomas Jefferson National Accelerator Facility, Virginia, USA

M. F. Smith

Thomas Jefferson National Accelerator Facility, Virginia, USA

S. Majewski

Thomas Jefferson National Accelerator Facility, Virginia, USA

See next page for additional authors

Follow this and additional works at: https://ro.uow.edu.au/engpapers

Part of the Engineering Commons

https://ro.uow.edu.au/engpapers/72

Recommended Citation

Meikle, S. R.; Wojcik, R.; Weisenberger, A. G.; Smith, M. F.; Majewski, S.; Kench, P.; Eberl, S.; Fulton, R. R.; Lerch, M. L.; and Rosenfeld, Anatoly B.: CoALA-SPECT: a coded aperture laboratory animal SPECT system for pre clinical imaging 2002.

https://ro.uow.edu.au/engpapers/72

Research Online is the open access institutional repository for the University of Wollongong. For further information contact the UOW Library: research-pubs@uow.edu.au 


\section{Authors}

S. R. Meikle, R. Wojcik, A. G. Weisenberger, M. F. Smith, S. Majewski, P. Kench, S. Eberl, R. R. Fulton, M. L. Lerch, and Anatoly B. Rosenfeld 


\title{
CoALA-SPECT: A Coded Aperture Laboratory Animal SPECT System for Pre Clinical Imaging
}

\author{
Steven R. Meikle, Senior Member IEEE, Randy Wojcik, Andrew G. Weisenberger, Mark F. Smith, Member \\ IEEE, Stan Majewski, Peter Kench, Stefan Eberl, Member IEEE, Roger R. Fulton, Senior Member IEEE, \\ Michael Lerch, Member IEEE and Anatoly B. Rosenfeld, Senior Member IEEE
}

\begin{abstract}
We have previously demonstrated the potential of multi-pinhole coded apertures when used in conjunction with compact pixelated detectors for high resolution, high sensitivity small animal SPECT. We are now constructing a prototype SPECT system with three such detectors mounted on a rotating gantry. Each detector comprises a $12 \mathrm{~cm}$ diameter circular array of $\mathrm{NaI}(\mathrm{Tl})$ crystals, each $1 \times 1 \times 5 \mathrm{~mm}^{3}$ on a $1.25 \mathrm{~mm}$ pitch. Each crystal array is coupled to a Hamamatsu $R 329212.7 \mathrm{~cm}$ diameter PS-PMT. Signals from the three detectors are read out by a custom subtractive resistive circuit and multiplexed. Data are acquired by a National Instruments PCI-6110E board running on a Macintosh dual processor G4 computer under Kmax software control. Images are reconstructed using a 3D iterative MAP-EM algorithm based on a multi-pinhole forward and back projector. The trade-off between contrast and variance was studied by simulation for a number of multipinhole configurations. We also performed initial planar imaging studies on one of the detectors. Our results indicate that spatial resolution approaching $1 \mathrm{~mm}$ full width at half maximum (FWHM) can be achieved with a single pinhole aperture, while improved contrast and variance may result from using optimized multi-pinhole apertures.
\end{abstract}

\section{INTRODUCTION}

$\mathrm{R}$ ecent advances in imaging instrumentation have led to a variety of tomograph designs for dedicated pre clinical imaging of laboratory animals. Applications include evaluation of promising new radiopharmaceuticals, study of the molecular origins of human disease and evaluation of new forms of therapy. These applications and advances in instrumentation are applicable to positron emitters and single photon emitters. PET is able to image a wide range of

This work was supported by the University of Sydney under a major equipment grant and by the Office of Biological and Environmental Research of the U.S. Department of Energy. The Southeastern Universities Research Association operates the Thomas Jefferson National Accelerator Facility for the U.S. Department of Energy under contract DE-AC05-84ER40150.

S. R. Meikle (telephone: 02-9515-6173, e-mail: steve@cs.usyd.edu.au), S. Eberl (stefan@cs.usyd.edu.au) and R. R. Fulton (rfulton@nucmed.rpa.cs.nsw.gov.au) are with the Department of PET and Nuclear Medicine, Royal Prince Alfred Hospital, Sydney, NSW 2050 Australia

R. Wojcik (wojcik@jlab.org), A. G. Weisenberger (drew@jlab.org), M. F. Smith (mfsmith@jlab.org) and S. Majewski (majewski@jlab.org) are with the Thomas Jefferson National Accelerator Facility, Newport News, VA 23606 USA.

P. Kench (p.kench@cchs.usyd.edu.au) is with the School of Medical Radiation Sciences, University of Sydney, Sydney, NSW Australia.

M. Lerch (mlerch@uow.edu.au) and A. B. Rosenfeld (anatoly@uow.edu.au) are with the Centre for Medical Radiation Physics, University of Wollongong, Wollongong, NSW Australia. biologically important compounds and drugs, while SPECT has the advantage of greater radiotracer availability and longer lived isotopes. However, most of the instrumentation developments have been directed towards PET while single photon imaging has, to date, mainly been confined to suitably modified clinical systems [1]. While very high spatial resolution can be achieved with this approach, it is limited by the vastly inferior detection efficiency compared with PET.

We have previously demonstrated the feasibility of using a multi-pinhole array as an encoding aperture, together with compact high resolution pixelated gamma detectors [2, 3]. This results in substantially increased detection efficiency compared with conventional SPECT systems while maintaining high spatial resolution. In this paper, we describe a prototype coded aperture SPECT system which is under construction. We also describe our image reconstruction methodology and results of initial planar imaging studies.

\section{DESIGN}

\section{A. Detectors}

Each detector comprises a $12 \mathrm{~cm}$ diameter disk of $5 \mathrm{~mm}$ thick $\mathrm{NaI}(\mathrm{Tl})$ crystal cut into 6140 pixels, each of which is $1 \times 1 \times 5 \mathrm{~mm}^{3}$ with a $1.25 \mathrm{~mm}$ centre to centre spacing (SaintGobain, Newbury $\mathrm{OH}$ ). The gaps between crystals are filled with aluminium oxide powder to optically isolate the crystals. The crystal array is glued to a $5 \mathrm{~mm}$ thick optical window of $\mathrm{K}^{+}$glass and encapsulated in a hermetically sealed unit. The sealed unit is attached to a Hamamatsu R3292 $12.7 \mathrm{~cm}$ diameter PS-PMT (Hamamatsu Photonics, Hamamatsu City, Japan) using a dry coupling to reduce spreading of the light. The detectors are contained in a telescopic unit comprising an inner cylinder of aluminium and an outer cylinder of $6 \mathrm{~mm}$ thick lead. A multi-pinhole aperture is positioned parallel to the detector face and the distance from the detector and source can be varied to achieve the desired spatial resolution, sensitivity and imaging field of view.

\section{B. Readout}

The R3292 is a crossed wire anode design with $28 \mathrm{X}$ and $28 \mathrm{Y}$ anode signals. The anode signals are converted to $(\mathrm{X}, \mathrm{Y})$ position signals by a custom subtractive resistive read-out circuit [4]. The anode wires from the PS-PMT are summed in pairs producing $14 \mathrm{X}$ and $14 \mathrm{Y}$ signals. These are amplified and the signals are split with half of each signal being fed to an inverting summing circuit. A fraction of the inverted 


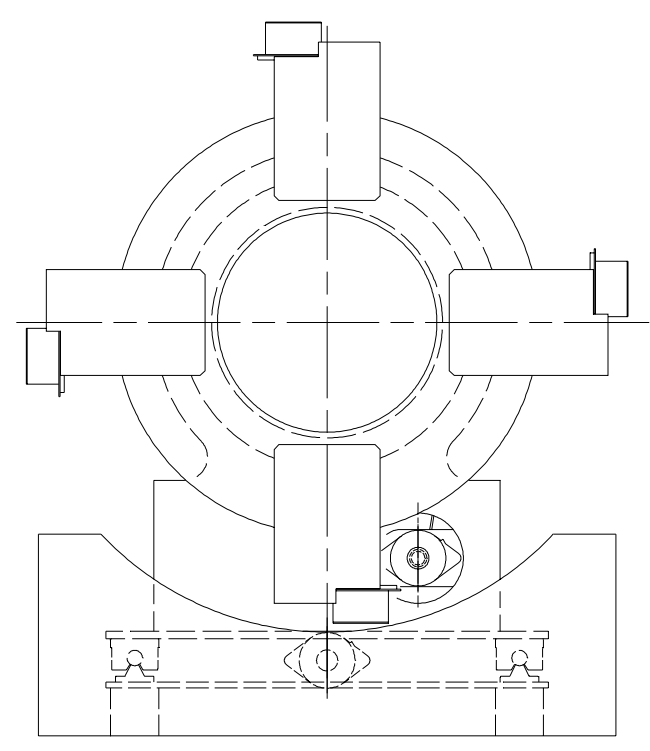

Fig. 1. Front view of CoALA-SPECT gantry with four detectors shown.

output is then fed to separate summing amplifiers for each $\mathrm{X}$ channel resulting in a scaled subtraction. The fraction to subtract is typically $5-7 \%$. The output of the subtraction circuit is fed to a charge division resistor chain which produces an $\mathrm{X}^{+}$and $\mathrm{X}^{-}$signal. These are fed to a 4 channel National Instruments PCI-6110E ADC board. The Y position signals are treated in the same way. The $\mathrm{X}$ sum signal is also used to trigger the DAQ board via a constant fraction discriminator. The signals from all detectors are multiplexed using a switching circuit. Data acquisition is controlled by a Kmax driver (Sparrow Corporation, Daytona Beach, FL) operating on a Macintosh dual processor G4 computer.

\section{Gantry Design}

The gantry is a custom designed table top unit with a rotating ring that supports the detectors and stepper motors (Fig. 1). The ring can support up to 4 detectors and the rotating trunnion is supported on linear guide rails. The gantry allows independent radial movement of the detectors, clockwise and counter clockwise rotation of up to 360 degrees with 0.5 degree precision and linear translation with $0.5 \mathrm{~mm}$ precision. All motions are controlled by a micro processor that communicates with the stepper motors over a miniature LAN via a single data cable.

\section{METHODS}

\section{A. Detector Calibration}

An image was obtained by acquiring data with approximately $40 \mathrm{MBq}$ of ${ }^{68} \mathrm{Ge} /{ }^{68} \mathrm{Ga}$ placed 1 metre from the detector. Data were acquired for several hours to ensure good statistics are obtained in each pixel ( $<1 \%$ standard deviation). A high energy $(511 \mathrm{keV})$ source is used to provide maximum light output from the crystals. The high count image was used

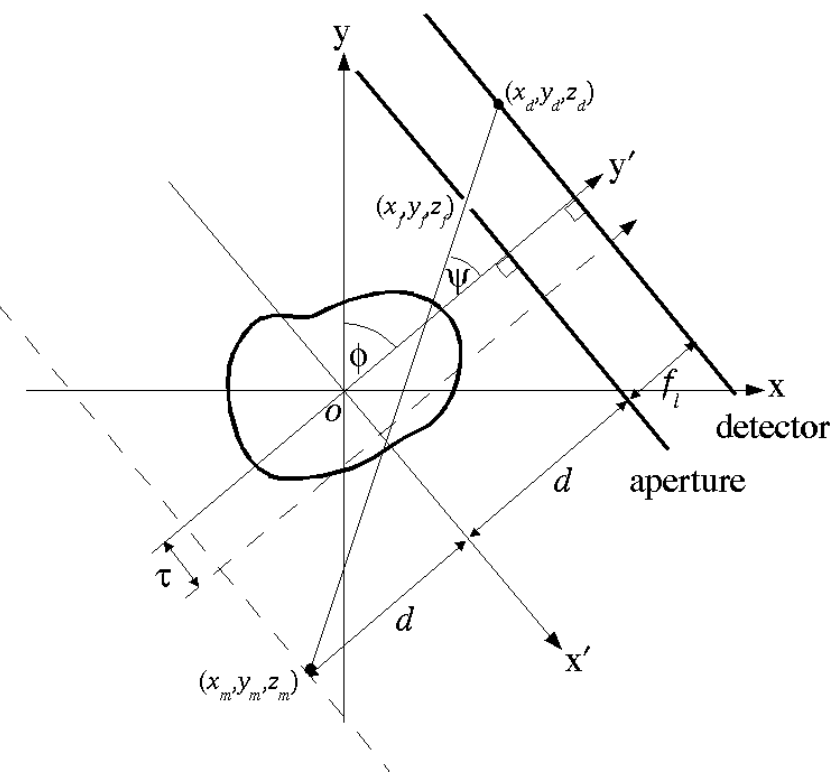

Fig. 2. Imaging geometry for a single pinhole projected onto the $x-y$ plane, with a transaxial component of displacement $\tau$ from the centre of rotation $O$. The displacement in the axial direction (into the page) is $\eta$.

to define the crystal boundaries. First, a semi-automated peak finding algorithm identified the locations of the crystal peaks. Then, lines were drawn in the valleys between crystal peaks using a fully automated algorithm. These lines define the crystal boundaries which are used by subsequent lookup tables to identify individual crystals.

For energy correction, data are acquired using a source of the same energy as that used for imaging. A $50 \mathrm{MBq}$ source of ${ }^{99 \mathrm{~m}} \mathrm{Tc}$ was placed 1 metre from the detector and data acquired for 2 hours. Energy spectra were acquired simultaneously for all pixels. At completion of the acquisition, an automated algorithm finds the photopeak channel in each spectrum and records this in an energy lookup table. During imaging, this lookup table is used to calculate the energy correction factor for each crystal and global upper and lower energy thresholds are applied. The image acquired for energy correction may also be used as an intrinsic flood for uniformity correction if acquired for sufficient time to reduce the pixel standard deviation below $1 \%$. The correction factor for each crystal is defined as the image mean divided by the individual pixel count.

\section{B. Image Reconstruction}

The approach to image reconstruction is similar to that described by $\mathrm{Li}$ et al. for single pinhole SPECT with a displaced centre of rotation [5]. We have extended the method to multiple pinholes, with each pinhole having a unique axial and transaxial displacement from the centre of the detector.

With reference to Fig. 2, the method (which is described in detail in [3]) is to determine the coordinates of each pinhole focal point and its corresponding mirror point in the fixed (unrotated) reference frame as 


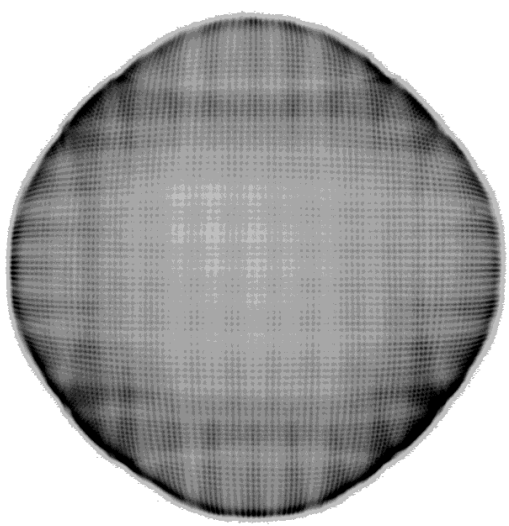

Fig. 3. Image of a $511 \mathrm{keV}$ point source recorded by the uncollimated detector.

$$
\begin{aligned}
& x_{f}=-d \sin \phi+(x+\tau) \cos \phi \\
& y_{f}=d \cos \phi+(x+\tau) \sin \phi \\
& z_{f}=z+\eta
\end{aligned}
$$

and

$$
\begin{aligned}
& x_{m}=d \sin \phi-\left(x_{d} \frac{2 d}{f_{l}}-x-\tau\right) \cos \phi \\
& y_{m}=-d \cos \phi-\left(x_{d} \frac{2 d}{f_{l}}-x-\tau\right) \sin \phi \\
& z_{m}=z+\eta-\left(z_{d}-z_{f}\right) \frac{2 d}{f_{l}}
\end{aligned}
$$

respectively, where $(x, z)$ is the displacement of the pinhole from the centre of the aperture on a plane parallel to the detector. These coordinates provide the start and end points which can be used with Siddon's ray tracing technique [6] to carry out the forward and back projection steps. The reconstruction is carried out using the iterative EM algorithm $[7,8]$ with MAP regularisation based on the one step late method [9].

\section{Simulations}

We performed simulations to evaluate the trade-off between contrast and variance when using the 3D ML-EM algorithm to reconstruct multi-pinhole data and to compare different multi-pinhole designs. The phantom used is shown in the first row of Fig. 5 . It is a $3 \mathrm{~cm}$ diameter cylinder with uniform activity apart from two small cylinders, one with $50 \%$ lower concentration than the background and one with $50 \%$ higher concentration. Pinhole arrays studied included one with a single pinhole at the centre of the field of view, and 2, 4, 6 and 8 pinholes placed on a regular grid with $1 \mathrm{~cm}$ spacing between them. Projections were generated at $20^{\circ}$ increments over $360^{\circ}$ and Poisson noise was added. Images were reconstructed and contrast and variance were calculated at each iteration. Contrast was defined as

$$
\left(\frac{\bar{C}_{\text {hot }}-\bar{C}_{\text {warm }}}{\bar{C}_{\text {hot }}+\bar{C}_{\text {warm }}}\right) x 100 \%
$$

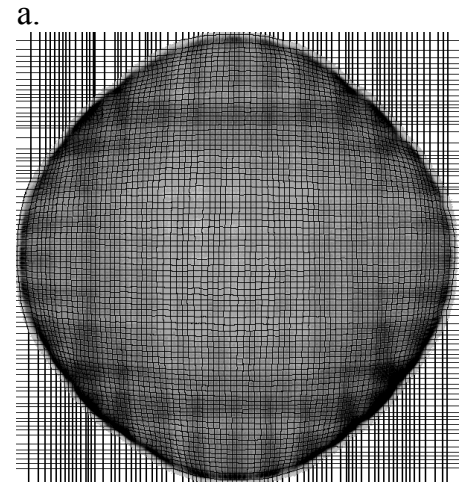

b.

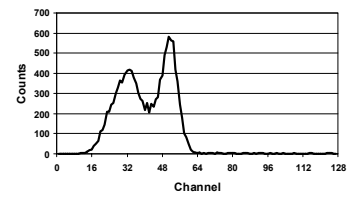

c.



Fig. 4. Crystal identification map (a) and individual energy spectra corresponding to a centre crystal (b) and an edge crystal (c).

where $\bar{C}_{\text {hot }}$ and $\bar{C}_{\text {warm }}$ are the mean pixel values in the hot and warm cylinders respectively. Thus, a maximum contrast value of $50 \%$ is possible for this phantom. Variance was calculated for a region of interest in the background compartment of the phantom.

\section{Phantom Study}

To assess the imaging performance of the detectors and provide a qualitative measure of planar resolution, a phantom comprising 4 capillary tubes was imaged. The tubes have an outer diameter of $1.5 \mathrm{~mm}$ and wall thickness of $100 \mu \mathrm{m}$. Three of the four tubes were filled with ${ }^{99 \mathrm{~m}} \mathrm{Tc}$ and the tubes were taped together so that they were touching, with the unfilled tube 2 nd from the end. The phantom was imaged with a $0.5 \mathrm{~mm}$ diameter double knife edge pinhole insert made of tungsten. The pinhole was placed in a $2.5 \mathrm{~mm}$ thick lead mask and positioned $3 \mathrm{~cm}$ from the phantom. The phantom was imaged with pinhole-to-detector distances of 3 , 6 and $9 \mathrm{~cm}$, resulting in magnification factors of 1,2 and 3 . Spatial resolution was measured by fitting a Gaussian function to the count profile of the rightmost capillary tube.

\section{RESUlts}

\section{A. Detector Calibration}

The image in Fig. 3 is the position histogram obtained from one of the detectors when exposed to a source of 511 $\mathrm{keV}$ photons. The image demonstrates the ability of the PSPMT and subtractive resistive read-out circuit to clearly delineate individual $1 \mathrm{~mm}$ crystals right to the edge of the field of view. It also demonstrates the inherently non-uniform gain and spatial non-linearity across the field of view of the PS-PMT. This image was used to generate a crystal lookup map (Fig. 4a). Typical individual pixel energy spectra from a centre and edge pixel are shown in Fig. 4b and Fig. 4c. The photopeak channel is found for each pixel and recorded in an energy lookup table which is used for energy correction and setting energy thresholds.

\section{B. Simulations}

Images obtained with a single pinhole and a 6 pinhole array are shown in Fig. 5 and the corresponding contrast- 


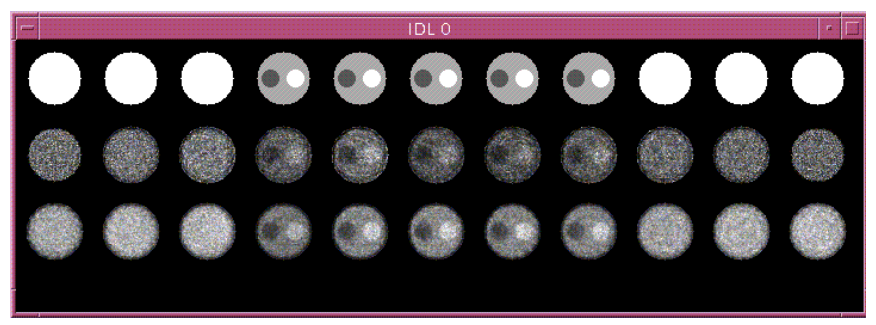

Fig. 5. Simulated contrast phantom (top row), reconstruction of the phantom using a single pinhole (middle row) and reconstruction using a 6 pinhole array (bottom row).

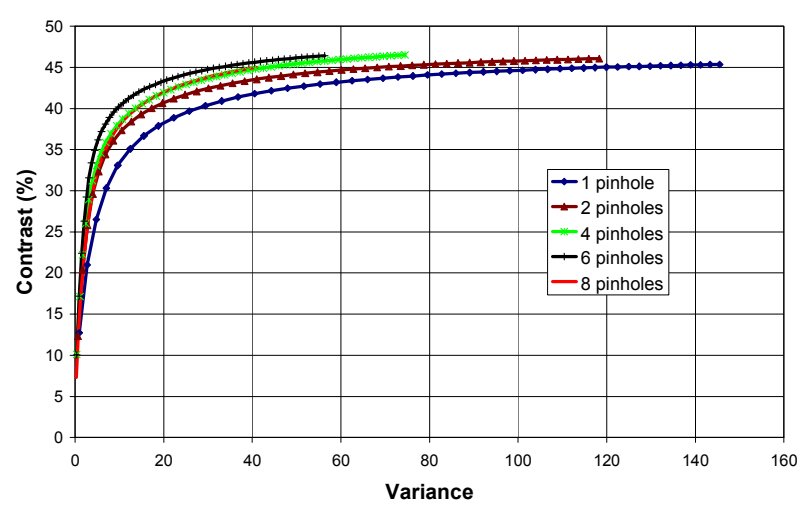

Fig. 6. Plots of contrast versus variance for various numbers of pinholes.

variance curves are shown in Fig. 6. Our results with this pinhole design and performance measure indicate that:

1. performance improves with increasing number of pinholes up to an optimum of 6 , and

2. the number of iterations required to reach an optimal point increases with increasing number of pinholes, i.e. convergence becomes slower.

Thus, while increasing the number of pinholes increases absolute sensitivity, it does not necessarily improve performance for a given imaging task. We expect that the optimal pinhole configuration will be object and task dependent. Further work is required to better understand the trade-offs with multi-pinhole reconstruction and to optimize their design.

\section{Phantom Study}

Images of the line source phantom are shown in Fig. 7. The $3^{\text {rd }}$ and $4^{\text {th }}$ line sources which are separated by the cold capillary tube are clearly delineated at all three levels of magnification. The $1^{\text {st }}$ and $2^{\text {nd }}$ line sources are also clearly delineated at a magnification factor of 3 and reasonably well delineated at a magnification of 2 but not at a magnification of 1 . These line sources are separated only by the thin walls of each capillary tube (approximately $200 \mu \mathrm{m}$ in total). The full width at half maximum (FWHM) of the count profiles (Fig. 8) was $2.15 \mathrm{~mm}, 1.25 \mathrm{~mm}$ and $0.95 \mathrm{~mm}$ for magnification factors of 1,2 and 3 respectively.

\section{DISCUSSION}

In this paper we described the design of our small animal SPECT scanner, image reconstruction methodology and some
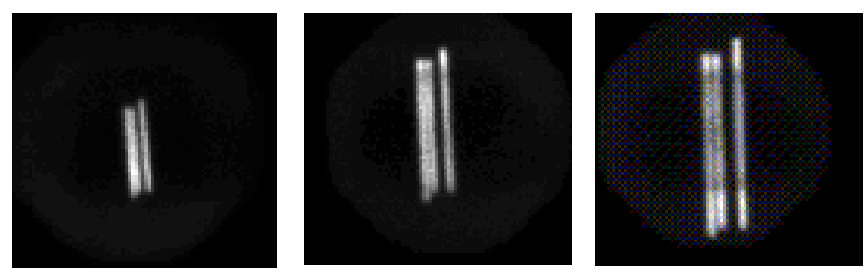

Fig. 7. Images of the line source phantom obtained with a single pinhole at magnification factors of 1 (left), 2 (centre) and 3 (right).
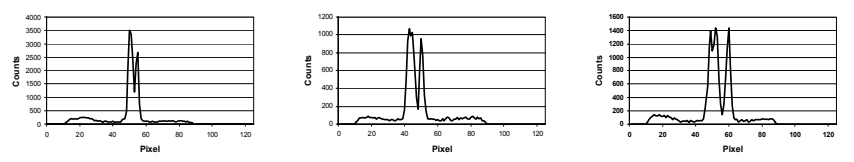

Fig. 8. Count profiles taken through the images of the line source phantom above.

preliminary imaging results. Our results indicate that the 1 $\mathrm{mm}$ crystals are well delineated by the PS-PMT and readout circuit and planar spatial resolution is between $1 \mathrm{~mm}$ and 2 $\mathrm{mm}$ FWHM depending on magnification. These results are very encouraging but further work is required to fully characterize the system and establish optimal pinhole configuration and reconstruction parameters.

Our gantry design will allow a minimum field of view of 5 $\mathrm{cm}$ in image space when the pinhole apertures are at the maximum range of their inward radial travel (i.e. when they touch at the vertices of the triangle formed). With the $12 \mathrm{~cm}$ field of view of our detectors, the maximum magnification at the centre of the field of view will be 2.5 . Thus, we can anticipate spatial resolution at the centre of approximately 1 $\mathrm{mm}$ FWHM for small source objects such as the mouse and the rat brain. For whole body imaging of the rat, a larger field of view diameter will result in less magnification and spatial resolution closer to $2 \mathrm{~mm}$ FWHM. The sensitivity of the device will also be dependent on magnification and the pinhole configuration employed. Our aim is to achieve an order of magnitude improvement in effective sensitivity over an equivalent single detector, single pinhole device.

In this study, we used a single tungsten pinhole insert placed in a lead mask to perform the initial imaging studies. Several multi-pinhole apertures have been designed and constructed based on the simulation results presented in this paper. The holes are $0.7 \mathrm{~mm}$ diameter double knife edge holes with $45^{\circ}$ acceptance angle and $1 \mathrm{~mm}$ channels drilled into $7 \mathrm{~mm}$ thick tungsten sheets. There are up to 6 pinholes per aperture. These apertures will help to determine the optimal multi-pinhole configuration and gain a better understanding of the various trade-offs for physical phantom data and how they affect performance of the imaging task.

\section{REFERENCES}

[1] D. A. Weber and M. Ivanovic, "Ultra-high-resolution imaging of small animals: implications for preclinical and research studies," J Nucl Cardiol, vol. 6, pp. 332-344, 1999.

[2] S. R. Meikle, R. R. Fulton, S. Eberl, M. Dahlbom, K.-P. Wong, and M. J. Fulham, "An investigation of coded aperture imaging for small animal SPECT," IEEE Trans Nucl Sci, vol. 48, pp. 816-821, 2001. 
[3] S. R. Meikle, P. Kench, A. G. Weisenberger, R. Wojcik, M. F. Smith, S. Majewski, S. Eberl, R. R. Fulton, A. B. Rosenfeld, and M. J. Fulham, "A prototype coded aperture detector for small animal SPECT," IEEE Trans Nucl Sci, vol. 49, pp. 2167-2171, 2002.

[4] R. Wojcik, S. Majewski, B. Kross, V. Popov, and A. G. Weisenberger, "Optimized readout of small gamma cameras for high resolution single gamma and positron emission imaging," presented at Conference Record of the 2001 IEEE Nuclear Science Symposium and Medical Imaging Conference, San Diego, J. A. Seibert, Ed., 2001.

[5] J. Li, R. J. Jaszczak, and R. E. Coleman, "Maximum likelihood reconstruction for pinhole SPECT with a displaced center-of-rotation," IEEE Trans Med Imag, vol. 14, pp. 407-409, 1995.

[6] R. L. Siddon, "Fast calculation of the exact radiological path for a three-dimensional CT array," Med Phys, vol. 12, pp. 252-255, 1985.

[7] L. A. Shepp and Y. Vardi, "Maximum likelihood reconstruction for emission tomography," IEEE Trans Med Imag, vol. MI-1, pp. 113$122,1982$.

[8] K. Lange and R. Carson, "EM reconstruction algorithms for emission and transmission tomography," J Comput Assist Tomogr, vol. 8, pp. 306-316, 1984.

[9] P. J. Green, "Bayesian reconstruction from emission tomography data using a modified EM algorithm," IEEE Trans Med Imag, vol. 9, pp. 84-93, 1990. 